\section{La aplicación de teorías y técnicas de las ciencias sociales a la promoción de la salud ${ }^{1}$}

Poco a poco se han ido incorporando en los programas de promoción de la salud y prevención de enfermedades varias teorías y técnicas que han resultado productivas en el campo de las ciencias sociales. En América del Norte y Europa han despertado interés especial las que se conocen, respectivamente, como etapas de cambio, aprendizaje social, organización comunitaria, propagación de innovaciones, psicología conductual de la comunidad, mercadeo social, modelo de cambio de comportamiento, y los modelos de cambio de comportamiento-comunicación I y II. También se han propuesto el modelo unificado y el de "preceder y proceder" (precede/proceed) como estructuras que combinan los diferentes modelos y teorías básicos en la planificación de programas de promoción de la salud.

Para que estas teorías de las ciencias sociales refuercen los programas comunitarios de prevención (1), deben cumplir con ciertas características. Tienen que ser fáciles de adoptar por todos los miembros de la comunidad y no solamente los más pudientes. Además, deben ser lo suficientemente flexibles para satisfacer las necesidades de grupos concretos, responder a nueva información y a las sugerencias emanadas de la comunidad interesada. El costo de los programas tiene que mantenerse bajo, de modo que no constituya una barrera para los más pobres. Por otra parte, los planificadores deben estar familiarizados con la cultura y los valores de la comunidad y tenerlos en cuenta al formular actividades; a la gente no le gusta adoptar comportamientos que llamen la atención. Por último, los miembros de la comunidad deben involucrarse en todos los aspectos de un programa para asegurarse de que satisface sus necesidades reales. Es más probable que un programa dé buenos resultados si es bien conocido en la comunidad. Los dirigentes y residentes de la comunidad deben estar involucrados en todas las fases de cada programa para que, en último término, este pueda entregarse a la comunidad como cosa suya.

\section{Etapas de cambio}

Según esta teoría que se formuló por observación directa de personas que estaban efectuando cambios en su comportamiento (2), lo más común es pasar por cinco etapas a medida que uno se familia- 
riza con una nueva conducta. Aplicadas, por ejemplo, a dejar de fumar, esas etapas serían las siguientes: 1) La persona todavía no ha pensado en cambiar su comportamiento ("Fumo muy poco".). 2) La persona empieza a contemplar algunos cambios ("Quizá me convenga abandonar el hábito".). 3) La persona se prepara para el cambio ("Mandé a pedir unos materiales que me van a ayudar a dejar de fumar".). 4) La persona empieza a efectuar cambios ("He pasado todo el día sin fumar".). 5) Dos o tres años después del cambio, la persona continúa comportándose de forma diferente ("Desde que dejé de fumar he ahorrado suficiente dinero para esas vacaciones que siempre he deseado tomar".). Las personas que desean cambiar algún comportamiento deben incorporarse a programas y servicios que comiencen con la etapa en que ellas se encuentran.

En este modelo se distinguen también diversos procesos que llevan al cambio de comportamiento. La concienciación es el proceso de proporcionar información a la población de forma que pueda responder de manera eficaz a los factores que la afectan. También ocurre el proceso de catarsis, que permite aliviar las emociones reprimidas y conduce a una mejor salud. Los cambios que los individuos deben llevar a cabo se facilitan mediante el manejo de contingencias (es decir, los resultados del comportamiento), procedimientos para controlar estímulos en el ambiente, y reevaluación o cambio de respuesta a un comportamiento sin alterar el ambiente.

El proceso de autoliberación permite a las personas familiarizarse con comportamientos optativos. El de liberación social modifica el comportamiento de grupos mediante cambios de política - como las leyes de equidad en el trabajo- que brindan más opciones.

\section{Aprendizaje social}

En esta teoría de contacto interpersonal (3), el comportamiento se define como producto de una serie de factores personales y ambientales y se transforma de acuerdo con los aspectos que se explican a continuación. En primer lugar, para estimular un comportamiento saludable, es preciso variar los factores externos del ambiente que rodea a la persona. Por ejemplo, es más fácil que una madre embarazada participe en actividades para la prevención del bajo peso al nacer si tiene acceso al cuidado gratuito de sus otros niños.

Las creencias de una persona con respecto a los comportamientos saludables son muy importantes. A veces es esencial impartir la información necesaria para corregir creencias equivocadas (por ejemplo, que la gente mayor no debe hacer ejercicio físico). Otras veces, para llegar a adoptar un com- portamiento sano, la persona requiere algún adiestramiento especial, como aprender a hacer un nuevo ejercicio. Debe tenerse en cuenta que las personas aprenden más fácilmente por observación. La imitación de personas modelo es una de las formas más efectivas de enseñar comportamientos saludables.

Por otra parte, también es cierto que las personas son más propensas a repetir un comportamiento sano si esperan recibir por ello una recompensa. La alabanza por algo logrado o bien hecho es una recompensa muy apreciada. Además, interesa más un comportamiento que se sabe traerá un resultado que se considera bueno, como por ejemplo, reducir de peso. Puesto que la confianza en uno mismo es la clave del éxito, los nuevos comportamientos deben introducirse paso a paso, de manera que las personas puedan desarrollar confianza en su habilidad para adoptarlos y mejorar su salud. Todo depende de una relación tripartita entre la persona, su comportamiento y el ambiente. El comportamiento no es solo el resultado de cómo la persona actúa y reacciona ante su ambiente, como tampoco es el ambiente el resultado de la persona y su comportamiento. Los tres factores están relacionados entre sí y las estrategias de cambio tienen que abarcarlos todos: ambiente, habilidades y cambio personal.

Para aplicar exitosamente la teoría del aprendizaje social, se recomienda que los promotores de salud establezcan un plan en tres fases (4). Primero deben especificar los resultados deseados (por ejemplo, en una escuela, reducir el número de alumnos que fuman). Luego deben identificar los principios que mejor puedan llevar a la población escolar a dejar de fumar. La creación de expectativas negativas sobre las consecuencias de fumar puede disuadir a los alumnos más pequeños de probar los cigarrillos, pero los más grandes, que ya fuman, a veces requieren programas de orientación que les ayuden a cultivar el control necesario para dejar de fumar. También es necesario parear las estrategias con los principios teóricos del aprendizaje social. Los planificadores tienen a su alcance el uso de estrategias - como el pronunciamiento de metas y el automonitoreo- que ayudan a los estudiantes a controlar su propio comportamiento y a dejar de fumar.

Antes de empezar el programa es importante asegurarse de que existen todas las condiciones previas necesarias para modificar el comportamiento: apoyos ambientales, habilidades, conocimientos y motivación. Los estudiantes de mayor edad pueden necesitar algo más que mayor fuerza de voluntad para dejar de fumar. Una posibilidad es la de los programas de cese del tabaquismo, en los cuales se trabaja poco a poco hasta que el estudiante adquiere la habilidad necesaria para abandonar el hábito. 
En las actitudes y los comportamientos de los adolescentes en particular ejercen mucha influencia los de sus iguales. El apoyo de los comportamientos sanos por parte del grupo social al que pertenecen tiene una gran influencia. En algunos programas que han dado muy buenos resultados participaron como consejeros o ayudantes otros jóvenes de la misma edad. En consecuencia, siempre debe procurarse que sean miembros de la comunidad quienes se encarguen de las actividades.

\section{Organización comunitaria}

Mediante esta técnica, los miembros de la comunidad reconocen los problemas comunes y pueden, en conjunto, movilizar recursos y elaborar y llevar a cabo los planes para resolverlos (5). Es una forma comprobada de hacer frente a problemas de salud que afectan a muchas personas.

Hay tres tipos principales de organización comunitaria (6). En el tipo 1, el de planificación social, se busca la ayuda de expertos externos para que traten de resolver los problemas de la comunidad junto con personas del lugar. Esos expertos recolectan datos sobre los problemas locales y recomiendan las vías de acción más razonables. Por lo general, en estos casos hay organismos formales que intervienen en la solución de los problemas y los educadores en sanidad se encargan de recolectar datos y de implementar y facilitar los programas. Es un proceso dirigido a tareas específicas.

En el tipo 2, el de desarrollo de la localidad, los expertos y los miembros de la comunidad trabajan juntos como iguales. Es un proceso orientado a impulsar el sentido de identidad comunal. Los educadores sanitarios organizan grupos representativos de toda la comunidad y los habilitan para señalar y resolver los problemas comunes. Este proceso hace hincapié en la cooperación y los educadores sirven de coordinadores y facilitadores, mientras instruyen a los miembros de la comunidad en valores y técnicas para resolver sus problemas.

El tipo 3, el de acción social, se centra tanto en el proceso como en las tareas. Está bajo el control de los miembros de la comunidad, quienes procuran adquirir la capacidad necesaria para abordar los problemas que más les interesa resolver. De este modo se pueden lograr cambios importantes y corregir el desequilibrio de poder que existe entre los grupos en situación desventajosa y el resto de la sociedad. La acción social suele tomar la forma de organizaciones y manifestaciones políticas de grandes grupos. Por lo general, un educador sanitario interesado en proyectos de acción social hace de activista, agitador y negociador. Esta teoría ha funcionado bien para grupos como los defensores del ambiente y la ecología, los pacifistas y el movimiento de derechos de la mujer.

En la teoría de organización comunitaria hay ciertas normas que se asocian con la capacidad de efectuar cambios para conseguir la salud. Una es que en todas las fases de cualquier programa de promoción de la salud participen miembros de la comunidad, con objeto de crear la sensación de posesión de algo propio. Un programa es mucho más efectivo cuando la comunidad se siente dueña de él (7). A veces es necesario adiestrar a algunos miembros de la comunidad para que adquieran los conocimientos y habilidades que necesitan para participar en las actividades o hacerse cargo de un programa. No es realista esperar una participación general sin ese adiestramiento. Si los planificadores del programa dan por sentado que los miembros de la comunidad tienen los conocimientos, las habilidades y el poder necesarios para involucrarse activamente en los programas, estos pueden sentirse abrumados e incapaces de actuar $(8,9)$.

La organización comunitaria es una forma eficaz de promover cambios ambientales que faciliten la adopción de comportamientos sanos. En Finlandia, por ejemplo, en el proyecto de salud cardíaca se trabajó con grupos de la comunidad y, como resultado, se prohibió fumar en sitios públicos.

\section{Propagación de innovaciones}

Esta teoría se basa en la noción de que es más fácil adoptar nuevas prácticas -incluso comportamientos saludables - si son similares a algo que la persona ya conoce; suficientemente flexibles para practicarse en diversas situaciones; fáciles de intentar y de comprender; reversibles o fáciles de interrumpir si se desea; mejores que otras posibles prácticas; de bajo riesgo; y de bajo costo, no solo en función de dinero, sino también de tiempo y esfuerzo (10).

Según esta teoría las personas se clasifican en una de seis categorías, de acuerdo con el tiempo que les toma adoptar nuevas ideas y prácticas. En una comunidad, los miembros que tienen mucha influencia se clasifican a menudo como adoptadores precoces. La influencia de estas personas puede persuadir a otros miembros de la comunidad a ensayar nuevas ideas o prácticas.

Esta teoría se ha criticado porque, si bien la suposición de que una persona adoptará innovaciones pone el interés en la persona antes que en la innovación, tal parece que la teoría trata de ajustar a la persona a la innovación en vez de lo contrario. Algunos investigadores consideran que no se han tenido en cuenta muchos factores que pueden afectar a la capacidad o al deseo de una persona de 
adoptar nuevas ideas o prácticas. Además se critica el aspecto elitista de que los comportamientos saludables se filtrarán poco a poco hacia abajo, a los grupos más necesitados.

Se ha sugerido que esta teoría puede dar mejores resultados si se buscan innovaciones que sean halagadoras para diferentes grupos. Puesto que la gente se siente más inclinada a probar comportamientos flexibles, reversibles, fáciles de ensayar, ventajosos, simples, efectivos en función de su costo y compatibles con sus valores, dentro de las actividades programáticas debe hacerse hincapié en los comportamientos sanos que tengan esas características (por ejemplo, caminar durante 15 minutos todos los días). Se ha recomendado también añadir campañas de mercadeo social para acelerar aun más la adopción de innovaciones en todos los grupos.

\section{Psicología conductual de la comunidad}

Según esta teoría, los programas pueden fundamentarse en las estrategias para el cambio de comportamiento propuestas por Elder et al. (11). Estas se basan en que la gente suele tener más interés en cambiar de conducta si a cambio de ello recibe una recompensa o puede esperar que suceda algo bueno. Es esencial hacer hincapié en los resultados positivos. Es mucho menos probable que cambien un comportamiento dañino cuando el resultado es desagradable. Sin embargo, pueden establecerse restricciones que desalienten un comportamiento indeseado; por ejemplo, la inspección al azar de automóviles en las vías públicas por parte de la policía desanima el manejo de vehículos en estado de embriaguez. Por el contrario, la reacción favorable de sus iguales motivará a un niño a seguir fumando.

De igual modo, hay barreras que impiden que ciertas personas adopten nuevos comportamientos saludables. Por ejemplo, puede que haya mujeres embarazadas que desean asistir a programas prenatales, pero no tienen con quién dejar a sus otros niños. Si se les resuelve ese problema, adoptarán la conducta apropiada de asistir al control prenatal.

Otra forma de alentar los comportamientos sanos y desalentar los que perjudican la salud es ofrecer retroinformación después de la medición de parámetros físicos como el peso o la tensión arterial. Debe procurarse que la mayoría de los miembros de la comunidad puedan fácilmente participar en todas las actividades de promoción de la salud. Es esencial que la comunidad pueda apreciar los buenos resultados y entenderlos fácilmente. Por ejemplo, puesto en un lugar público, un cartel que muestre claramente la disminución del número de fumadores en un pueblo puede generar suficiente entusiasmo para que otros se unan al proyecto.
Los programas deben funcionar en muchos niveles a la vez. Su éxito depende de que refuercen el comportamiento de individuos, grupos, organizaciones y comunidades simultáneamente (12). Así, muchas personas pueden modificar su conducta individual mientras participan en actividades programáticas a gran escala.

\section{Mercadeo social}

Esta teoría fue definida (13) como "el diseño, la implementación y el control de programas que buscan aumentar la aceptabilidad de una idea o práctica social en un grupo definido". Usualmente entraña el uso de los medios de comunicación - como periódicos, folletos, radio y televisiónpara poner al público al tanto de nuevas ideas y prácticas, incluidos los comportamientos saludables. No obstante, el mercadeo social es algo más que simple propaganda, pues determina lo que un grupo específico de personas necesita, imparte información para que las personas tomen decisiones fundamentadas, les ofrece programas o servicios para satisfacer sus necesidades y evalúa cuántas de estas han sido satisfechas.

El mercadeo social descansa en una serie de conceptos:

- Intercambio. Los individuos o grupos intercambian dinero o esfuerzos por algún material, servicio o idea si están convencidos de que los beneficios - por ejemplo, el dinero que ahorran dejando de fumar- valen la pena.

- Orientación del consumidor. El mercadeo social se concentra en la audiencia, es decir, en el consumidor. Esto constituye una de sus principales ventajas y su mayor aporte a cualquier programa (14).

- Análisis de la audiencia. Al principio hay que reconocer lo que necesitan los miembros de la comunidad. Seguidamente se determinan los costos y beneficios de satisfacer esas necesidades.

- Segmentación de la audiencia. Los miembros de la comunidad se agrupan según sus similitudes (características y necesidades) para que los planificadores puedan diseñar los mensajes y programas que cada grupo necesita. En ese análisis y segmentación de la audiencia (15) se toman en consideración la geografía, demografía (edad, género, tamaño de la familia, raza) y estructura social (lugares de trabajo, escuelas, organizaciones religiosas, grupos de voluntarios, gobiernos locales).

- Integración del mercadeo. En todas las actividades del mercadeo social se consideran el producto que se promueve, el precio (pagadero en dinero, tiempo o cambio de estilo de vida), la promoción 
(métodos de comunicación y mercadeo social), la situación (posicionamiento de los mensajes de la campaña) y la participación del propio consumidor.

La mayor virtud del mercadeo social es que llega a un gran número de personas. Al emplearlo, los planificadores llegan a una comprensión más profunda de la población objetivo (16). Sin embargo, sin otras estrategias de apoyo, su capacidad para cambiar los comportamientos es limitada. El mercadeo social por sí solo es una estrategia de poco impacto y aun las campañas de mercadeo social más eficaces aumentan el logro de los objetivos del programa solo en 15\%.

Otra desventaja del mercadeo social es el costo de elaborar, ejecutar y evaluar campañas que involucran publicidad. En el campo de la salud, especialmente, es difícil competir con la propaganda comercial de compañías que a menudo promueven comportamientos perjudiciales. Además, el mercadeo social no trata adecuadamente con conductas complejas, no ayuda a la gente a comprender asuntos de salud complicados, ni enseña cómo manejarse en situaciones tan difíciles como la presión del grupo de iguales.

\section{Modelo de cambio de comportamiento}

Este modelo se puso en práctica en Finlandia para desarrollar todos los programas y servicios que formaron parte del proyecto de salud del corazón ya mencionado (17). Se basa en la idea de que la gente adopta nuevos comportamientos cuando ve que otras personas ya lo han hecho. Es un tipo de aprendizaje observacional que ocurre cuando una persona nota que otras reciben recompensas y respaldo por cierto comportamiento. También incluye elementos de la teoría de campo clásica (18) y la de acción razonada $(19,20)$. Esta última implica que las acciones de una persona dependen primordialmente de sus intenciones conductuales o deseo de comportarse de cierta manera. La fuerza de ese deseo depende de la actitud de la persona hacia la conducta y la influencia de normas subjetivas generales. Como la teoría de aprendizaje social, la de acción razonada reconoce que otras personas y normas sociales pueden influir mucho en la adopción de un nuevo comportamiento.

El modelo del cambio de comportamiento se ha usado para explicar los comportamientos relacionados con la salud de distintas culturas. Según Kar et al. (21), las intenciones, el respaldo social de personas importantes y el acceso a los conocimientos y servicios afectan al comportamiento en torno a la salud en los diversos medios culturales. Los proyectos de promoción de salud que siguen este modelo pueden guiarse por los principios siguientes, utilizados en el programa finlandés (17).

- Mejora de los servicios. Se reorganizaron los servicios de salud locales y se establecieron programas de tamizaje para identificar mejor a las personas en riesgo de cardiopatías.

- Información. Para concienciar a la gente sobre la relación entre su comportamiento y la salud, se llevó a cabo una gran campaña de información pública en una variedad de medios, entre ellos la radio y los periódicos.

- Persuasión. Se procuró que todos los mensajes elaborados para el proyecto fueran muy convincentes.

- Adiestramiento y apoyo social. Como parte del proyecto, se realizaron talleres para enseñar comportamientos saludables que pudieran ser difíciles de aprender, por ejemplo, la preparación de comidas sanas para mantener la salud cardiovascular. Todos estos programas siguieron cuatro etapas básicas: demostración (modelado) del comportamiento; práctica dirigida del nuevo comportamiento; recomendaciones o retroinformación sobre cómo mejorar la ejecución del comportamiento; y ofrecimiento de apoyo social para alentar a las personas a perseverar en él.

- Cambios en el ambiente. El propósito de esas acciones es quitar las barreras y crear oportunidades para el cambio de comportamiento. El proyecto estimuló a las organizaciones de la comunidad a adoptar políticas para proteger la salud cardiovascular; por ejemplo, se prohibió fumar en sitios públicos para crear un ambiente local que fomentara los comportamientos sanos para el corazón.

- Organización de la comunidad. Miembros clave de la comunidad respaldaron el proyecto y tomaron parte en las actividades.

\section{Modelos de cambio de comportamiento-comunicación}

Estos modelos se usaron en dos proyectos diferentes de salud del corazón $(17,22,23)$, para planear programas y campañas de información pública. Los dos modelos incluyeron los ocho elementos siguientes:

- Concienciación. Concienciar a las personas de los problemas de salud y posibles soluciones.

- Atención. Seleccionar una audiencia objetivo apropiada para la campaña y los métodos para comunicarse con ella, por ejemplo, mediante avisos por televisión. 
- Motivación. Hacer hincapié en la importancia de la fuente del mensaje. Es más probable que las personas cambien cuando saben cuáles son las cosas buenas que pueden ocurrir como resultado.

- Conocimiento. La información sobre comportamientos saludables debe ser fácil de comprender, interesante y útil para los miembros de la comunidad. Los mensajes deben estar a tono con la comunidad para la cual se han diseñado. Es decir, deben reflejar las formas de hablar y vestirse de los miembros de la comunidad.

- Cambio de actitud. Entre los métodos para cambiar de actitud figuran la participación de dirigentes de la comunidad, el contacto más frecuente con los miembros de la comunidad y el desarrollo de actividades apropiadas.

- Habilidades. Con frecuencia hay que adiestrar a las personas para que aprendan nuevos comportamientos, aun cuando son difíciles. Estos deben enseñarse mediante demostraciones y prácticas. Además, es importante brindar retroinformación a las personas que están tratando de aprender esos comportamientos.

- Desempeño. Puede alentarse la ejecución de los nuevos comportamientos ofreciendo retroinformación, actividades comunitarias especiales, respaldo social, adiestramiento dirigido a aumentar la autoconfianza y ayuda práctica.

- Mantenimiento. Las actividades que conducen a cambios ambientales saludables, como el respaldo social, el adiestramiento para aumentar la autoconfianza y los trabajos de organización comunitaria, estimulan a la población a mantener los nuevos comportamientos saludables.

\section{Modelo unificado}

En uno de los proyectos finlandeses (17) se combinaron la teoría de organización comunitaria, el modelo de cambio de comportamiento, los ocho aspectos del modelo de cambio de comportamiento-comunicación y la teoría de propagación de innovaciones, en un solo entramado que se llamó el modelo unificado. Este modelo se usó más bien como fundamento de todo el proyecto y no para planear actividades específicas.

\section{Modelo "preceder y proceder"}

En este modelo, las teorías anteriormente descritas pueden emplearse como estructura de un modelo general para planificar programas de promoción de la salud. A continuación se describen las nueve fases de este modelo y las teorías que corresponden a la ejecución de cada fase.
Las fases 1 y 2 tienen una íntima relación mutua y se dedican al diagnóstico social y al epidemiológico. Partiendo de la base de que los problemas sociales de una comunidad son una medida práctica y exacta de la calidad de vida (24), la fase 1 requiere reconocer sus necesidades, carencias y problemas. Se determina así la calidad de vida en esa localidad. La fase 2 tiene como objeto determinar las metas de salud y las dificultades y problemas de salud que se relacionan con los hallazgos de la primera fase. En este proceso pueden utilizarse: las teorías de la organización comunitaria, para darles a los residentes locales la oportunidad de reconocer los problemas que comparten; el mercadeo social, que determina las necesidades de un grupo prioritario; la teoría de aprendizaje social, para identificar los factores ambientales que conducen a comportamientos perjudiciales; y la propagación de innovaciones, para determinar las necesidades o problemas de la comunidad.

En la etapa 3 se buscan las causas ambientales o los comportamientos que podrían estar relacionados con los problemas hallados en la etapa 2. Con esos fines pueden ponerse en práctica aspectos de las teorías de etapas de cambio para determinar en qué etapas del comportamiento están los grupos de población; de aprendizaje social, para determinar la capacidad de comportamiento, las expectativas, la respuesta emocional y otras cualidades de la población objetivo; de propagación de innovaciones, para determinar en qué etapa de la adopción de innovaciones está la población, y de mercadeo social, para que se aplique el principio de la segmentación.

La etapa 4 es de diagnóstico educativo y organizativo. Se dedica a clasificar en tres categorías los comportamientos y factores ambientales notados en la etapa 3: factores que estimulan o inhiben los cambios de comportamiento; barreras que impiden el cambio o las habilidades y conocimientos necesarios para cambiar; y recompensas o mensajes que recibe la gente y sus efectos en cuanto a estimular o desalentar la ejecución de algún comportamiento a largo plazo. En este proceso pueden ser útiles las teorías de aprendizaje social, organización comunitaria, propagación de innovaciones, psicología conductual de la comunidad, mercadeo social, el modelo de cambio de comportamiento y los modelos de cambio de comportamiento-comunicación.

La etapa 5 comprende una búsqueda de recursos que puedan utilizarse para emprender un programa, por ejemplo, los canales de comunicación. Las teorías de mercadeo social y de organización comunitaria son apropiadas en esta etapa.

Durante la etapa 6 se ejecuta el programa.

Durante las etapas 7 a 9, el programa se repasa para evaluar su funcionamiento, impacto y resultados. 


\section{SYNOPSIS}

\section{Theories and methods of the social sciences as applied to health promotion}

We briefly describe herein those social science theories and methods that have slowly been incorporated into health promotion and disease prevention programs. They include the various stages of behavior change, social learning, commu- nity organization, spread of innovations, social marketing, and others. Such theories can be very useful for planning and implementing community programs as long as these are low in cost, have enough flexibility to satisfy the needs of target groups, respond to new information and to the suggestions of community members, show sensitivity to the local culture, and involve representatives from the community in all of their activities.

\section{REFERENCIAS}

1. Lefebvre RC, Lasater TM, Carleton RA, Peterson G. Theory and delivery of health programming in the community: The Pawtucket Heart Health Program. Prev Med 1987;16:80-95.

2. Rimer BK. Perspectives on intrapersonal theories in health education and health behaviour. En: Glanz K, Lewis FM, Rimer BK, eds. Health behavior and health education: Theory, research, and practice. San Francisco: Jossey-Bass; 1990; pp. 140-158.

3. Bandura A. Social learning theory. Englewood Cliffs, New Jersey: Prentice-Hall 1990.

4. Perry $\mathrm{C}$, et al. How individuals, environments and health behavior interact: Social learning theory. En: Glanz K, Lewis FM, Rimer BK, eds. Health behavior and health education: Theory, research, and practice. San Francisco: Jossey-Bass; 1990. pp. 161-186.

5. Minkler M. Improving health through community organization. En: Glanz K, Lewis FM, Rimer BK, eds. Health behavior and health education: Theory, research, and practice. San Francisco: Jossey-Bass; 1990. pp. 257-287.

6. Rothman J, Tropman JE. Models of community organization and macro practice: their mixing and phasing. En: Cox FM, Erlich JL, Rothman J, Tropman JE, eds. Strategies of community organization. 4a ed. Itasca, Illinois: Peacock; 1987. pp. 3-26.

7. Pancer SM, Nelson G. Community-based approaches to health promotion. Toronto: Ontario Ministry of Health; 1990.
8. Hunt S. Building alliances: professional and political issues in community participation: Examples from a health and community development project. Health Promot Int 1990;5:179-185.

9. Piette D. Community participation in formal decision-making mechanisms. Health Promot Int 1990;5:187-197.

10. Rogers EM. Diffusion of innovations. 3a ed. New York: Free Press; 1983.

11. Elder JP, Hovell MF, Lasater TM, Wells BL, Carleton RA. Applications of behaviour modification to community health education: The case of heart disease prevention. Health Educ O 1985;12: 151-168.

12. Minkler M. Improving health through community organization. En: Glanz K, Lewis FM, Rimer BK, eds. Health behavior and health education: Theory, research, and practice. San Francisco: Jossey-Bass; 1990. pp. 169-181.

13. Kotler P. Marketing for nonprofit organizations. Englewood Cliffs, New Jersey: Prentice-Hall; 1982.

14. Novelli WD. Applying social marketing to health promotion and disease prevention. En: Glanz K, Lewis FM, Rimer BK, eds. Health behavior and health education Theory, research, and practice. San Francisco: Jossey-Bass; 1990. pp. 342-369.

15. Lefebvre RC, Flora JA. Social marketing and public health intervention. Health Educ Q 1988;15:299-315.

16. Mintz J. Social marketing: New weapon in an old struggle. Health Promot 1988; $27: 6-12$
17. Puska P, Nissinen A, Tuomilehto J, Salonen JT, Koskila K, McAlister A, et al. The community-based strategy to prevent coronary heart disease: Conclusions from the ten years of the North Karelia project. Annu Rev Public Health 1985;6: 147-193.

18. Lewin K. Field theory in social science: Selected theoretical papers. New York: Harper and Row; 1951.

19. Fishbein M, Ajzen I. Beliefs, attitudes, intention, and behaviour: An introduction to theory and research. Reading, Massachussetts: Addison-Wesley;1975.

20. Ajzen I, Fishbein M. Understanding attitudes and predicting social behavior. Englewood Cliffs, New Jersey: Prentice-Hall; 1980.

21. Kar SB, et al. A psychosocial model of health behaviour: Implications for nutrition education, research and policy. Health Values 1983;7:29-37.

22. Farquhar JW, et al. The Stanford fivecity project: Designs and methods. En: Matarazzo JD. Behavioral health: A handbook of health enhancement and disease prevention. New York: John Wiley and Sons; 1984.

23. Shea S, Basch CE. A review of five major community-based cardiovascular disease prevention programs: Part 1, Rationale, design, and theoretical framework. Am J Health Promot 1990:4:203-213.

24. Green LW, Kreuter MW. Health promotion planning: An educational and environmental approach. 2a ed. Mountain View, California: Mayfield; 1991. 